\section{Zum Einfluß einer genaueren Strömungsmodellierung auf die Beschreibung von Festbettreaktoren ${ }^{*}$}

\section{Thomas Daszkowski und Gerhart Eigenberger**}

Herrn Professor Dr-Ing. Karl Stephan zum 60. Geburtstag

Zur Modellierung und Berechnung von katalytischen Festbettreaktoren gibt es eine große Anzahl von Arbeiten. Übersichtsbeiträge $[1,3]$ zeigen Möglichkeiten der Klassifizierung dieser Modelle und deren Einsatzbereich. Dabei ergibt sich, daß bereits recht einfache Modelle das qualitative Verhalten technischer Reaktoren zutreffend beschreiben. Für eine zutreffende quantitative Beschreibung müssen aber in der Regel sowohl die kinetischen Parameter als auch die Parameter des Wärmetransports an die experimentellen Ergebnisse angepaßt werden. Die Extrapolierbarkeit solcher Modelle auf andere Reaktorkonfigurationen ist damit deutlich eingeschränkt. Ein Grund für die mangelnde Extrapolierbarkeit wird in der unvollständigen Beschreibung der Strömungs- und Wärmetransportvorgänge gesehen. Arbeiten von Vortmeyer und Mitarb. [4] und anderen Arbeitsgruppen [5, 6] haben sich bereits mit dieser Frage befaßt.

Ziel der vorliegenden Mitteilung ist es, die in den vorstehend genannten Arbeiten benutzten Modellvorstellungen weiterzuentwickeln und ihre Aussagen experimentellen Ergebnissen gegenüberzustellen.

* Überarbeitete Fassung eines Vortrags von T. Daszkowski auf der GVC-Fachausschußsitzung ,Technische Reaktionsführung", 5. bis 7. März 1990 in Hamburg.

** Dipl.-Ing. Th. Daszkowski und Prof. Dr.-Ing. G. Eigenberger, Institut für Chemische Verfahrenstechnik der Univ. Stuttgart, Böblinger Str. 72, 7000 Stuttgart 1.

\section{Modell des katalytischen Festbettreaktors}

Schüttungsgefüllte Festbettreaktoren zeichnen sich bei detaillierter Betrachtung durch eine große Uneinheitlichkeit der lokalen Durchströmung und des lokalen Wärme- und Stofftransports aus. Deswegen existiert eine lange und noch ungeklärte Diskussion darüber, ob das Reaktorverhalten mit kontinuierlichen Modellen oder Zellenmodellen beschrieben werden soll. Im folgenden wird der kontinuierlichen Modellierung der Vorzug gegeben, unter anderem deswegen, weil alle bisherigen Zellenmodelle eine reguläre Anordnung voraussetzen, die der Zufallsschüttung widerspricht.

\subsection{Strömungsmodellierung}

Die Geschwindigkeitsverteilung resultiert aus der gegebenen Hohlraumverteilung der Schüttungskörper. Die bei der Umströmung einzelner Katalysatorpartikeln auftretenden Beschleunigungen in axialer und radialer Richtung sowie Verwirbelungen werden bei der kontinuierlichen Beschreibung gemittelt. Die Strömungsmodellierung kann daher nur einen summarischen Charakter haben, der sich auf die Haupteinflüsse beschränkt.

Für durchströmte Schüttungen einheitlicher Hohlraumverteilung hat sich eine vereinfachte Impulsbilanz mit einem Druckverlustansatz nach Ergun bewährt. Sie wird im folgenden auch für eine örtlich veränderliche Hohlraumverteilung übernommen. In eindimensionaler Form lautet sie: ${ }^{1)}$

$\frac{\partial p}{\partial z}=-f_{1} v_{z}-f_{2} v_{z}^{2}$

1) Eine Zusammenstellung der Formelzeichen befindet sich am Schluß des Beitrags. 
mit den Faktoren:

$f_{1}=150 \mu \frac{(1-\varepsilon(r))^{2}}{\varepsilon(r)^{3} d_{\mathrm{p}}^{2}}, f_{2}=1,75 \varrho \frac{(1-\varepsilon(r))}{\varepsilon(r)^{3} d_{\mathrm{p}}}$

Sie beschreibt die Abhängigkeit des axialen Druckverlustes als Funktion der Leerrohrgeschwindigkeit und der örtlich veränderlichen Hohlraumverteilung $\varepsilon(r)$. In der globalen Betrachungsweise beinhaltet sie den Druckverlust, der sich aus der Reibung des Fluids an der Schüttungsoberfläche sowie der permanenten Beschleunigung und Verzögerung des Gases bei der Umströmung der Schüttungspartikel ergibt. Schwierigkeiten bereitet dabei der Übergang zur Rohrwand (Punktkontakt, Hohlraumanteil gegen 1), da die Beziehung nach Ergun nur für Hohlraumverteilungen kleiner 0,6 gültig ist. In einer empirischen Erweiterung ergänzte Brinkmann [7] die Ergun-Gleichung um einen Wandreibungsterm nach Navier-Stokes:

$\frac{\partial p}{\partial z}+f_{1} v_{z}+f_{2} v_{z}|v|-\mu\left[\frac{\partial^{2} v_{z}}{\partial z^{2}}\right]-\mu\left[\frac{\partial^{2} v_{z}}{\partial r^{2}}+\frac{1}{r} \frac{\partial v_{z}}{\partial r}\right]=0$

Diese Vorgehensweise läßt einige Fragen offen, weil die Reibung an der Rohrwand und die Reibung an den umströmten Partikeln in unterschiedlicher Weise beschrieben werden. Sie besitzt aber den großen Vorteil, in beiden Grenzfällen (Schüttung einheitlicher Porosität ohne Wandeinfluß und leeres Rohr) jeweils die richtige Gleichung zu liefern. Bei der Lösung der Gl. (2) zeigt sich, daß im Kernbereich der Schüttung die Terme nach Ergun deutlich größer (Faktor 50) als der Reibungsterm sind. Im Wandbereich überwiegt der Reibungsterm nach Navier-Stokes, da die Terme nach Ergun gegen Null laufen. In einem kleinen Übergangsbereich, Hohlraumanteil von $(0,6$ bis 0,8$)$, ergeben sich durch die Überlagerung offensichtlich Ungenauigkeiten.

Aufbauend auf obige Überlegungen wird in dieser Arbeit die zweidimensionale Navier-Stokes-Gleichung mit der zweidimensionalen Darstellung des Reibungsansatz nach Ergun verknüpft. Für die Gültigkeit in den Übergangsbereichen, Einlauf und Auslauf (Abb. 1) sowie dem Randbereich gilt die vorstehend getroffene Einschränkung

Die vollständigen Gleichungen zur Lösung der Geschwindigkeitsfelder sind in Massenstromdichten $G_{\mathrm{z}}, G_{\mathrm{r}}$ formuliert und beinhalten den jeweiligen Druckverlust, die Terme nach Ergun sowie die Beschleunigungs- und Reibungsterme nach Navier-Stokes. Als Lösungen folgen das Druckfeld und die axialen und radialen Massenstromgeschwindigkeiten. Bei den Massenströmen handelt es sich im Bereich der Schüttung um Zwischenkorn-Geschwindigkeiten. Stellvertretend ist nachstehend nur die axiale Impulsbilanz aufgeführt:
Leerrohr in die Schüttung. Mit der so gegebenen Hohlraumverteilung in axialer und radialer Richtung ist das durchströmte Gebiet charakterisiert.

Exemplarisch seien in Abb. 3 die berechneten Strömungsvorgänge im Einlaufbereich der Schüttung dargestellt. Berechnet wurde ein Rohr mit $50 \mathrm{~mm}$ Durchmesser, gefüllt mit $8 \mathrm{~mm}$ Kugeln. Die Länge

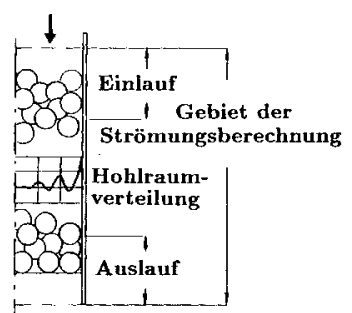

Abb. 1. Schematische Darstellung eines schüttungsgefüllten Rohres und der radialen Hohlraumverteilung.
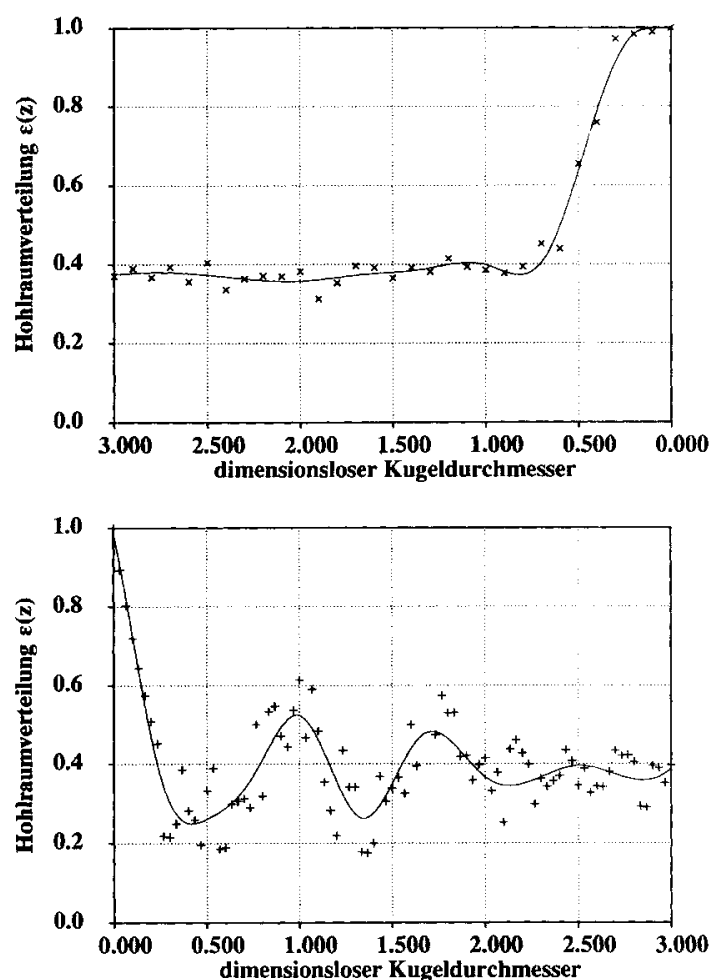

Abb. 2. Experimentell ermittelte axiale Hohlraumverteilung im Einlauf (oben) und Auslauf (unten).

$\frac{\partial p^{2}}{\partial z}+g_{1} \varepsilon G_{z}+g_{2} \varepsilon^{2} G_{z}|G|+2 \frac{R}{M}\left\{G_{z}\left[\frac{\partial\left(T G_{z}\right)}{\partial z}\right]+G_{\mathrm{r}}\left[\frac{\partial\left(T G_{z}\right)}{\partial r}\right]-\mu\left[\frac{\partial^{2}\left(T G_{z}\right)}{\partial z^{2}}\right]-\mu\left[\frac{\partial^{2}\left(T G_{z}\right)}{\partial r^{2}}+\frac{1}{r} \frac{\partial\left(T G_{z}\right)}{\partial r}\right]\right\}=0$

mit den Faktoren:

$g_{1}=\frac{R T}{M}\left[300 \mu \frac{(1-\varepsilon)^{2}}{\varepsilon^{3} d_{\mathrm{p}}^{2}}\right], g_{2}=\frac{R T}{M}\left[3,5 \frac{(1-\varepsilon)}{\varepsilon^{3} d_{\mathrm{p}}}\right]$

Zur Berechnung der Strömung ist es folglich notwendig, neben der bereits aus der Literatur bekannten radialen Hohlraumverteilung [8] zusätzlich die axiale Verteilung zu bestimmen. Abb. 2 zeigt die in eigenen Untersuchungen bestimmte Verteilung im Ein- und Auslaufbereich. $\mathrm{Zu}$ erkennen ist, daß im Auslaufbereich die aus der radialen Verteilung bekannten Schwingungen auftreten. Sie sind durch das Gitter, auf dem die Schüttung aufliegt, bedingt. Im Einlauf erfolgt ein nahezu schwingungsfreier Übergang vom des Rohres beträgt $600 \mathrm{~mm}$. Die Schüttung beginnt bei $150 \mathrm{~mm}$. Deutlich zu sehen ist die gleichmäßige Verteilung der axialen Massenstromdichte (Belastung) im Leerrohr. Die Ausprägung der Randgängigkeit erfolgt sehr rasch und im wesentlichen innerhalb einer Kugellage. Der Massenstrom wird insgesamt zusätzlich in axialer Richtung beschleunigt, da durch die Schüttung der freie Querschnitt verengt wird. Die radiale Komponente zeigt den Massentransport vom Rohrzentrum in den Wandbereich, der auf einen schmalen Bereich am Schüttungseintritt beschränkt ist.

\subsection{Konzentrations- und Temperaturfelder}

Zur Beschreibung der Konzentrations- und Temperaturfelder werden die Material- und Energiebilanzen eines örtlich zweidi- 
mensionalen Modells benutzt. Die darin auftretenden örtlich veränderlichen $G_{\mathrm{z}^{-}}$und $G_{\mathrm{r}}$-Werte folgen aus den beiden Impuls-

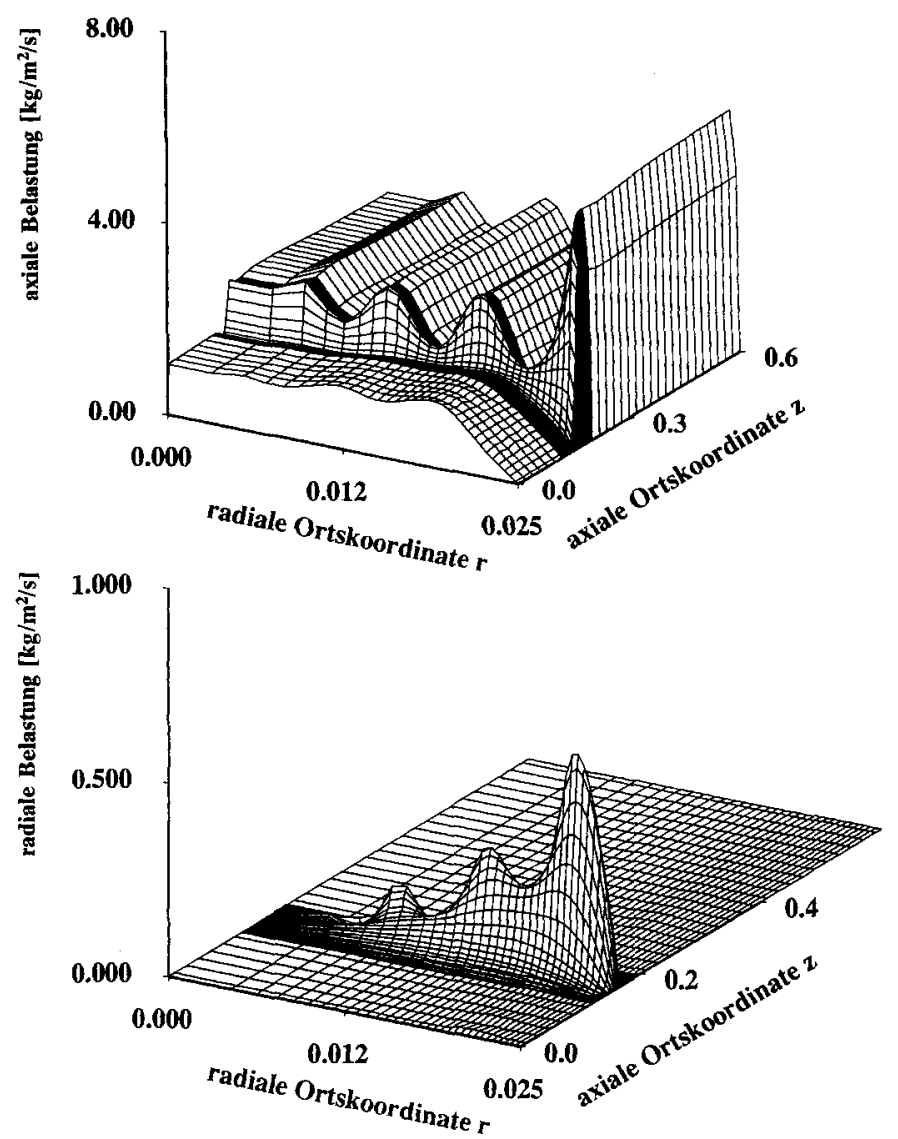

Abb. 3. Berechneter axialer (oben) und radialer (unten) Massenstrom im Einlauf.
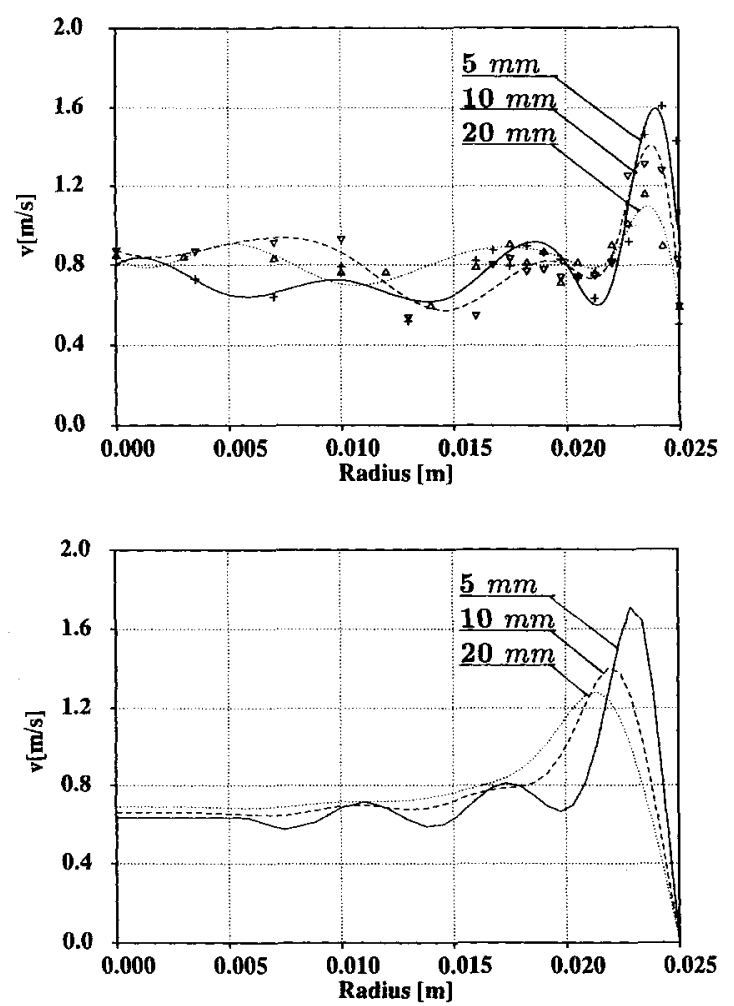

Abb. 4. Vergleich Experiment (oben) und Berechnung (unten) der axialen Strömungskomponente im Auslauf. bilanzen und der Kontinuitätsgleichung. Stellvertretend ist im folgenden die Materialbilanz für die Komponente $j$ aufgeführt:

$$
\begin{aligned}
\varepsilon \varrho^{\mathrm{G}} \frac{\partial w_{\mathrm{j}}}{\partial t}= & -G_{\mathrm{z}} \frac{\partial w_{\mathrm{j}}}{\partial z}-G_{\mathrm{r}} \frac{\partial w_{\mathrm{j}}}{\partial r}+\varepsilon \varrho^{\mathrm{G}} D_{\mathrm{jz}} \frac{\partial^{2} w_{\mathrm{j}}}{\partial z^{2}}+\varepsilon \varrho^{\mathrm{G}} D_{\mathrm{jr}} \times \\
& \times\left[\frac{\partial^{2} w_{\mathrm{j}}}{\partial r^{2}}+\frac{1}{r} \frac{\partial w_{\mathrm{j}}}{\partial r}\right]+M_{\mathrm{j}} \sum_{i=1}^{l} v_{\mathrm{ij}} r_{\mathrm{i}}\left(w_{\mathrm{n}}, T\right)
\end{aligned}
$$

\section{Experimentelle Untersuchung und Vergleich mit der berechneten Strömung}

Da Geschwindigkeitsmessungen mit Hitzdrahtanemometersonden innerhalb der Schüttung nicht möglich sind, wurde in verschiedenen Abständen oberhalb und unterhalb einer Kugelschüttung (Stahlkugeln mit $8 \mathrm{~mm}$ Durchmesser) gemessen. Dabei wurden entlang einer Umfangslinie mit dem Radius $r$ bis zu 16 zeitlich gemittelte Einzelmessungen der axialen Strömungskomponente aufgenommen. Die Mittelung dieser Werte ergibt die axiale Geschwindigkeit an der Stelle $r$. Abb. 4 gibt die gemessenen Profile 5, 10 und $20 \mathrm{~mm}$ unterhalb der Schüttung wieder. Experiment und Simulation zeigen deutlich, daß die Randgängigkeit im Auslauf nur langsam abgebaut wird. Das Profil direkt unterhalb der Schüttung (Abstand $5 \mathrm{~mm}$ ) spiegelt die Schwingung der radialen Hohlraumverteilung wieder. Zusammenfassend kann gesagt werden, daß Modell und Experiment qualitativ gut übereinstimmen.

\section{Einfluß der Strömungsvorgänge auf den Wärmetransport}

Zur Bestimmung der Wärmetransportparameter werden experimentelle Daten überwiegend mit Pfropfenströmungsmodellen ausgewertet. Im folgenden soll diskutiert werden, welche Unterschiede sich bei der Auswertung mit einem Pfropfenströmungsmodell und dem vorgestellten Modell ergeben. Die ungleichmäßige Strömungsverteilung, hohe Geschwindigkeit im Wandbereich und niedrige Geschwindigkeit im Kernbereich, bedingt unterschiedliche Verweilzeiten. Das Pfropfenströmungsmodell vernachlässigt dies. Daher wird der Kernbereich zu langsam und der Wandbereich zu schnell aufgeheizt. Bei der Anpassung der Simulation an das Experiment ergeben sich folglich je nach Modell unterschiedliche Werte für die Transportkoeffizienten. Das Modell mit Randgängigkeit liefert geringere Werte für den Wandwärmeübergangskoeffizienten wie für die radiale Wärmeleitfähigkeit. Beispielhaft seien (s. Tab. 1) für eine mittlere Leerrohrgeschwindigkeit von $1 \mathrm{~m} / \mathrm{s}$ die Wertepaare für 8 -mm-Keramikkugeln und $5-\mathrm{mm}$-Keramikstränge angegeben. Dabei bezieht sich jeweils der erste Wert auf die Auswertung mit dem Pfropfenströmungsmodell.

Außerdem erlaubt die vollständige Modellierung das Phänomen der in der Literatur diskutierten $[9,10]$,längenabhängigen Parameter" zu erläutern. Über das Modell läßt sich die eingetragene Wärmestromdichte als Funktion der Länge berechnen. Wie bereits angesprochen wird durch die Annahme der Pfropfenströmung der Wandbereich zu schnell erwärmt, so daß die Wärmestromdichte einen niedrigen Maximalwert annimmt. Will man mit dem Modell der Pfropfenströmung den Verlauf der Wärmestrom-

Tabelle 1. Wärmetransportparameter aus experimentellen Messungen, ausgewertet mit dem Pfropfenströmungsmodell und der detaillierten Modellvorstellung.

\begin{tabular}{lcc}
\hline & Kugel & Strang \\
\hline Wandwärmeübergangskoeffizient $\left[\mathrm{W} / \mathrm{m}^{2} \mathrm{~K}\right]$ & $160 / 96$ & $150 / 110$ \\
\hline Wärmeleitfähigkeit $[\mathrm{W} / \mathrm{m} \mathrm{K}]$ & $1,1 / 0,7$ & $1,5 / 1,1$ \\
\hline
\end{tabular}


dichte des vollständigen Modells beschreiben, ist man gezwungen, die Wärmetransportkoeffizienten im Einlaufbereich zu erhöhen.

\section{Einfluß der Strömungsvorgänge auf den Wärmetransport bei Ablauf chemischer Reaktion}

Bei dem reinen Wärmetransportproblem wurde gezeigt, daß Modellungenauigkeiten durch Anpassung der Wärmetransportparameter kompensiert werden können. Dies gilt im Falle des Wärmetransports bei Ablauf chemischer Reaktion nur bedingt. Im vorliegenden Fall wird die Totaloxidation von Propen an einem Edelmetall-Katalysator betrachtet. Die kinetischen Daten entstammen experimentellen Untersuchungen. Die Wärmetransportparameter entsprechen den Werten nach Tab. 1. Es wird keine weitere Anpassung vorgenommen, sondern gezeigt, wie sich mit diesen Parametern ein Versuchslauf bei einer Einlauf- und Kühltemperatur von $160^{\circ} \mathrm{C}$ beschreiben läßt. Die Konzentration an
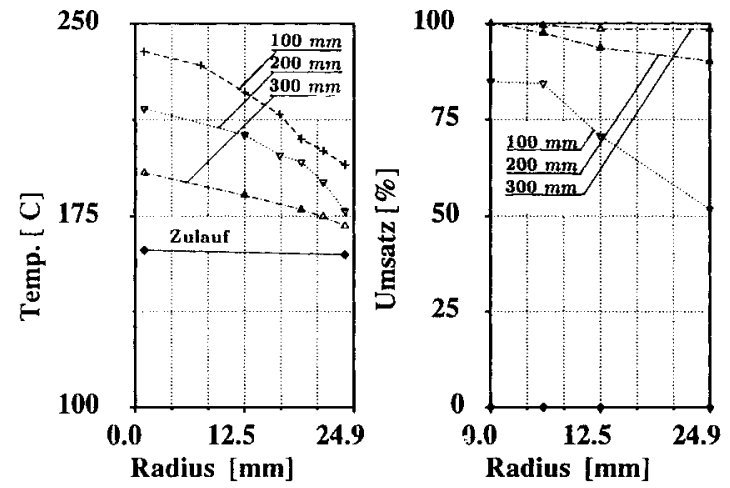

Abb. 5. Experimentell ermittelte Temperatur- und Konzentrationsverläufe für die Totaloxidation von Propen.
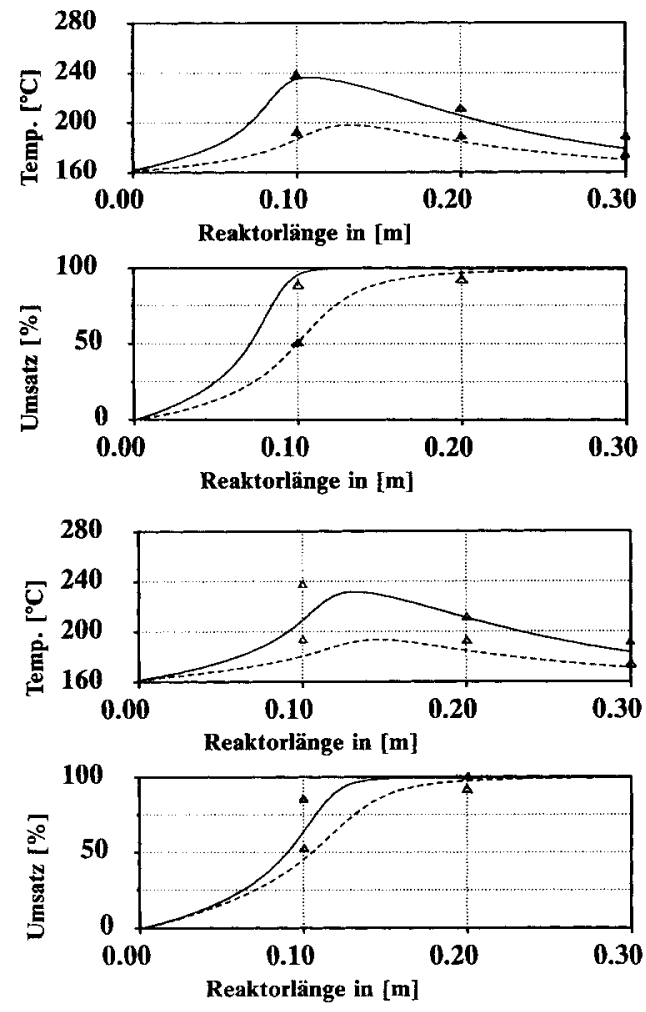

Abb. 6. Berechnete axiale Temperatur- und Konzentrationsverläufe in Rohrzentrum und -wand im Vergleich zu den Meßdaten; oben: vollständiges Modell, unten: Pfropfenströmungsmodell. - Rohrzentrum, ...- Rohrwand.
Propen im Zulauf beträgt $0,002 \mathrm{~kg}_{\mathrm{pr}} / \mathrm{kg}_{\mathrm{ges}}$. Der Versuchsaufbau ermöglicht die Messung von radialen Konzentrations- und Temperaturprofilen an den axialen Positionen 100, 200,300, $400 \mathrm{~mm}$ nach Reaktoreinlauf. Abb. 5 zeigt die gemessenen Konzentrations- und Temperaturprofile im Einlauf und an den Positionen 100, 200 und $300 \mathrm{~mm}$.

Das unterschiedliche Verweilzeitverhalten in der Schüttung bedingt, daß die Reaktion vom Rohrzentrum (hohe Verweilzeit) aus zündet. Die Temperatur im Rohrzentrum steigt rasch an, so daß in der Folge auch Propen im Wandbereich oxidiert wird. Wie das Experiment zeigt, besteht ein großes Konzentrationsgefälle zwischen Rohrwand und -zentrum. Das Modell mit randgängiger Strömung gibt das typische Reaktionsverhalten gut wieder. In Abb. 6 (oben) sind jeweils berechnete Temperatur- und Konzentrationsverläufe im Rohrzentrum und an der Rohrwand dargestellt. Zusätzlich sind die experimentellen Werte an den entsprechenden Meßebenen eingetragen. Bei der Berechnung des Reaktionsablaufs mit Pfropfenströmung (Abb. 6, unten) zündet die Reaktion später, da die Strömungsgeschwindigkeit im Rohrzentrum zu hoch angesetzt ist. Weder die Temperatur- noch die Konzentrationsverläufe werden, insbesondere in der ersten Meßebene, wiedergegeben. Das einfache Pfropfenströmungsmodell reicht nicht aus, um das Reaktorverhalten zu beschreiben. Eine Modellanpassung der kinetischen und der Wärmetransportparameter ist notwendig. Die eingangs angesprochenen Extrapolierbarkeit des Modells ist offensichtlich nicht gewährleistet.

Eingegangen am 1. Juni 1990 [K 1170]

\section{Formelzeichen}

$\begin{array}{lll}d_{\mathrm{T}} & {[\mathrm{m}]} & \text { Partikeldurchmesser } \\ D_{\mathrm{jr}} & {\left[\mathrm{m}^{2} / \mathrm{s}\right]} & \text { radialer Diffusionskoeffizient } \\ D_{\mathrm{jz}} & {\left[\mathrm{m}^{2} / \mathrm{s}\right]} & \text { axialer Diffusionskoeffizient } \\ G_{\mathrm{r}} & {\left[\mathrm{kg} /\left(\mathrm{m}^{2} \mathrm{~s}\right)\right]} & \text { radiale Massenstromdichte } \\ G_{\mathrm{z}} & {\left[\mathrm{kg} /\left(\mathrm{m}^{2} \mathrm{~s}\right)\right]} & \text { axiale Massenstromdichte } \\ M & {\left[\mathrm{~kg} / \mathrm{mol}^{2}\right]} & \text { Molmasse } \\ p & {\left[\mathrm{~N} / \mathrm{m}^{2}\right]} & \text { Druck } \\ r & {[\mathrm{~m}]} & \text { radiale Koordinate } \\ R & {[\mathrm{~J} /(\mathrm{mol} \mathrm{K})]} & \text { universelle Gaskonstante } \\ r_{\mathrm{i}} & {\left[\mathrm{mol} /\left(\mathrm{m}^{3} \mathrm{~s}\right)\right]} & \text { Reaktionsgeschwindigkeit } \\ T & {[\mathrm{~K}]} & \text { Temperatur } \\ v_{\mathrm{z}} & {[\mathrm{m} / \mathrm{s}]} & \text { axiale Geschwindigkeit } \\ w_{\mathrm{j}} & {\left[\mathrm{kg} / \mathrm{kg}_{\mathrm{ges}}\right]} & \text { Massenanteil } \\ z & {[\mathrm{~m}]} & \text { axiale Koordinate } \\ \varepsilon & {[-]} & \text { Hohlraumanteil } \\ \varrho & {\left[\mathrm{kg} / \mathrm{m}^{3}\right]} & \text { Dichte } \\ v_{\mathrm{jj}} & {[-]} & \text { stöchiometrischer Koeffizient } \\ \mu & {[\mathrm{kg} /(\mathrm{m} \mathrm{s})]} & \text { dynamische Viskosität }\end{array}$

\section{Literatur}

[1] Hofmann, H.: Chem.-Ing.-Tech. 51 (1979) S. 257/265.

[2] Vortmeyer, D.: Wärme Stoffübertrag. 21 (1987) S. 247/257.

[3] Eigenberger, G.: 7th. International Symposium Large Chemicals Plants, Bruges, 6.-7. Oct. 1988.

[4] Vortmeyer, D.; Schuster, J.: Chem. Eng. Sci. 38 (1983) S. $1691 / 1699$

[5] Venneti, M.; Govindarao, H.; Froment, G. F.: Chem. Eng. Sci. 41 (1986) S. 533/539.

[6] Ziolkowska, I.; Ziolkowski, D.: Chem. Eng. Process. 23 (1988) S. 137/164.

[7] Brinkmann, H. C.: Appl. Sci. Res. Sect. A1 (1947) S. 27.

[8] Benenati, R. F., Brosilow, C.: AIChEJ. 8 (1962) S. $359 / 361$.

[9] Hennecke, F. W.: Dissertation, Univ. Karlsruhe 1972.

[10] De Wasch, A. P.; Froment, G. F.: Chem. Eng. Sci. 27 (1972) S. $567 / 576$.

[11] Haidegger, E.; Vortmeyer, D.; Wagner, P.: Chem.-Ing.-Tech. 61 (1989) S. 647/650. 